\title{
Price Linked Order Quantity under Weibull Deterioration, with Quadratic Demand Rate without Salvage
}

\author{
Dr. R. Mohan \\ College of Military Engineering, F-Civil, Pune - 411031, India.
}

\begin{abstract}
This paper presents the possible effects of a temporary price discount offered by a supplier on a retailer's replenishment policy quadratic demand rate for the deteriorating items without salvage value. This model is developed by using the differential equation $\frac{d(I(t))}{d t}+\theta(t) I(t)=-f(t)$ when inventory items with decay process. where $I(t)$ is inventory level at time ' $t$ ', $f(t)$ is demand rate at time ' $t$ '. $\theta(t)$ is the decay rate. This paper presents quadratic demand with Weibull rate of deterioration without salvage value. In this paper, a study has been carried out to investigate the possible effects of a temporary price discount on a retailer's replenishment policy for deteriorating items. Salvage value is not considered in this case. Suitable numerical examples presented using MathCAD software. This paper investigates the decision process to assists retailers in deciding whether to adopt regular order policy special order policy. For the case if special order policy is adopted, the optimal order quantity for the retailers is determined by maximizing the total cost saving between special and regular order during special order period.
\end{abstract}

Keywords: Inventory, Deterioration, Quadratic demand, perishable, Weibull distribution

\section{Introduction}

Researchers developed inventory models generally consider two factors. These two factors of the problem have been of increasing interest are (i) deterioration of items and (ii) variations in demand rates. Each factor again is classified into two categories viz., (i) constant deterioration/ demand and (ii) time dependent deterioration/ demand. Earlier researchers Covert and Philip (1973) studied an EOQ model for items with weibull distributed deterioration. Buzacott (1975) developed an Economic order quantity with inflation Donaldson suggested inventory replenishment policy for a linear trend in demand. Dave and Patel (1981) proposed $\left(\mathrm{T}, \mathrm{S}_{\mathrm{i}}\right)$ - policy for inventory model for deteriorating items with time proportional demand. Misra (1975) studied optimum production lot size model for a system with deteriorating inventory. For a comprehensive review of literature on inventory models with time-varying demand and deteriorating items, one may refer the work of Giri and Goyal (2001). It is known that the demand for spare parts of new aero planes, advanced computer chips etc. increase rapidly while the demands for spares parts of the obsolete aero planes, computers etc. decrease very rapidly with time. This type of phenomena can well be addressed by inventory models with quadratic demand rate. Jalan et al. proposed EOQ model for items with Weibull distribution deterioration. They incorporated shortages in the inventory system. Bhandari and Sharma (2000) investigate a Single Period Inventory Problem with Quadratic Demand Distribution under the Influence of Marketing Policies. Khanra and Chaudhuri (2003) have discussed an order-level inventory problem with the demand rate represented by a continuous quadratic function of time. Sana and Chaudhuri (2004) suggested a stock-review inventory model for perishable items with uniform replenishment rate and stock-dependent demand. Mehta et al. (2006) investigates order-level lot size model for deteriorating items with exponentially deceasing demand. An order level EOQ model for deteriorating items in a single warehouse system with price depended demand in non-linear (quadratic) form has been studied by Patra et al (2010). Kalam et al (2010) have studied the problem of production lot-size inventory model for Weibull deteriorating item with quadratic demand, quadratic production and shortages. An economic order quantity model for items having linear demand under inflation and permissible delay in payments studied by Singh (2011). Mohan (2014) developed inventory model with quadratic demand, constant deterioration, variable holding cost with shortages and salvage value. Mohan (2014) proposed inventory system Weibull deterioration, quadratic demand under inflation. Thus an attempt is made, to investigate the decision process to assists retailers in deciding whether to adopt regular order policy special order policy. For the case if special order policy is adopted, the optimal order quantity for the retailers is determined by maximizing the total cost saving between special and regular order during special order period. 


\section{Assumptions}

The mathematical model is developed on the following assumptions and notations:

(i) The selling rate $D(t)$ at time $t$ is assumed to be $D(t)=a t^{2}+b t+c, a \neq 0, b \neq 0, c \geq 0$. Here $c$ is the initial rate of demand, $b$ is the rate with which the demand rate increases and $a$ is the acceleration of demand.

(ii) The deterioration rate follows two parameter Weibull distribution and if given by $\theta(t)=\alpha \beta t^{\beta-1} ; 0 \leq \alpha$ $\leq 1, \beta \geq 1$.

(iii) Replenishment rate is infinite and lead time is zero.

(iv) $\mathrm{C}$, the cost per unit

(v) $Q(t)$ is the inventory level at time $t$.

(vi) $A_{l}$ is the order cost per unit order.

(vii) $\mathrm{H}$ is the holding cost per unit

(viii) $\delta$ is the price discount rate

(ix) $\mathrm{q}$ is the order quantity under regular order policy

(x) $\mathrm{T}^{*}$ is the length of replenishment cycle time under regular policy

(xi) $\theta(t)=\alpha \beta t^{\beta-1}$ is the two parameter Weibull distribution rate

\section{Formulation and Solution of the Model}

The objective of the model is to determine the minimum total cost for items having time dependent quadratic demand, time dependent deterioration rate and salvage value.

The inventory level depletes as the time passes due to selling rate and deterioration. The differential equation which describes the inventory level at time $\mathrm{t}$ can be written as

$$
\begin{aligned}
& \frac{d Q(t)}{d t}+\theta(t) Q(t)=-\left(a t^{2}+b t+c\right) ; \theta=\alpha \beta t^{\beta-1} \quad 0 \leq t \leq T \\
& \text { with } Q(t)=0 \quad \text { at } t=\mathrm{T} .
\end{aligned}
$$

Equation (5.1) is a linear first order equation which can be written as

$$
\left(Q(t) e^{\alpha t^{\beta}}\right)^{\prime}=-\left(a t^{2}+b t+c\right) e^{\alpha t^{\beta}}
$$

which on integration yields

$$
Q(t) e^{\alpha t^{\beta}}=-\left(\frac{a t^{3}}{3}+\frac{b t^{2}}{2}+c t\right)-\alpha\left(\frac{a t^{\beta+3}}{\beta+3}+\frac{b t^{\beta+2}}{\beta+2}+\frac{c t^{\beta+1}}{\beta+1}\right)+k_{1}
$$

where $k_{1}$ is an integral constant. Here we have expanded $e^{\alpha t^{\beta}}$ and ignored higher order terms as $\alpha$ is small. The solution of the above differential equation using the boundary conditions is given by

$$
Q(t)=\left\{\begin{array}{l}
\frac{a}{3}\left(T^{3}-t^{3}\right)+\frac{b}{2}\left(T^{2}-t^{2}\right)+c(T-t) \\
+\alpha\left[\begin{array}{l}
\frac{a}{\beta+3}\left(T^{\beta+3}-t^{\beta+3}\right)+\frac{b}{\beta+2}\left(T^{\beta+2}-t^{\beta+2}\right) \\
+\frac{c}{\beta+1}\left(T^{\beta+1}-t^{\beta+1}\right)
\end{array}\right]
\end{array}\right\} e^{-\alpha t^{\beta}}
$$

Since $Q(0)=q$, we get

$$
q=\left(\frac{a T^{3}}{3}+\frac{b T^{2}}{2}+c T\right)+\alpha\left[\frac{a T^{\beta+3}}{\beta+3}+\frac{b T^{\beta+2}}{\beta+2}+\frac{c T^{\beta+1}}{\beta+1}\right]
$$


The following costs are calculated to find the total profit of the system when shortages are not allowed:

Ordering cost, $(\mathrm{OC})=\mathrm{A}_{1}$

Holding

cost,

(HC)

$h\left[\left(\frac{a T^{4}}{4}+\frac{b T^{3}}{3}+\frac{c T^{2}}{2}\right)+\alpha\left(\frac{a T^{\beta+4}}{(\beta+4)}+\frac{b T^{\beta+3}}{(\beta+3)}+\frac{c T^{\beta+2}}{(\beta+2)}\right)\left(\frac{\beta}{(1+\beta)}\right)\right]$

Material cost per cycle

(including Deterioration Loss) $=Q C$

Thus the total cost is obtained as

Total Cost $(\mathrm{TC})=$ Ordering cost + Holding cost + Material cost

$$
\begin{aligned}
& =\left\{\begin{array}{l}
\frac{h}{T}\left[\left(\frac{a T^{4}}{4}+\frac{b T^{3}}{3}+\frac{c T^{2}}{2}\right)+\alpha\left(\frac{a T^{\beta+4}}{(\beta+4)}+\frac{b T^{\beta+3}}{(\beta+3)}+\frac{c T^{\beta+2}}{(\beta+2)}\right)\left(\frac{\beta}{(1+\beta)}\right)\right] \\
+\frac{A}{T}+\frac{C}{T}\left[\left(\frac{a T^{3}}{3}+\frac{b T^{2}}{2}+c T\right)+\alpha\left(\frac{a T^{\beta+3}}{\beta+3}+\frac{b T^{\beta+2}}{\beta+2}+\frac{c T^{\beta+1}}{\beta+1}\right)\right]
\end{array}\right\} \\
& =\left\{\begin{array}{l}
h\left[\left(\frac{a T^{3}}{4}+\frac{b T^{2}}{3}+\frac{c T^{1}}{2}\right)+\alpha\left(\frac{a T^{\beta+3}}{(\beta+4)}+\frac{b T^{\beta+2}}{(\beta+3)}+\frac{c T^{\beta+1}}{(\beta+2)}\right)\left(\frac{\beta}{(1+\beta)}\right)\right] \\
+\frac{A}{T}+C\left[\left(\frac{a T^{2}}{3}+\frac{b T}{2}+c\right)+\alpha\left(\frac{a T^{\beta+2}}{\beta+3}+\frac{b T^{\beta+1}}{\beta+2}+\frac{c T^{\beta}}{\beta+1}\right)\right]
\end{array}\right\}
\end{aligned}
$$

From the above equation the unique value of $\mathrm{T}$, optimal length of replenishment cycle time ( say $\mathrm{T}^{*}$ ) can be obtained. Similarly the optimal order quantity $\mathrm{Q}^{*}$ can be found out in from $\mathrm{I}(\mathrm{t})$. i.e., $\mathrm{Q}(0)=\mathrm{q}$. The purpose of this paper is to study optimal order quantity by maximizing the total cost saving during the length of depletion time for the special order quantity.

Special order occurs (Retailers replenishment)

If the retailer order $\mathrm{Q}_{\mathrm{sp}}$ units under special order policy, the inventory level at time ' $\mathrm{t}$ ' is

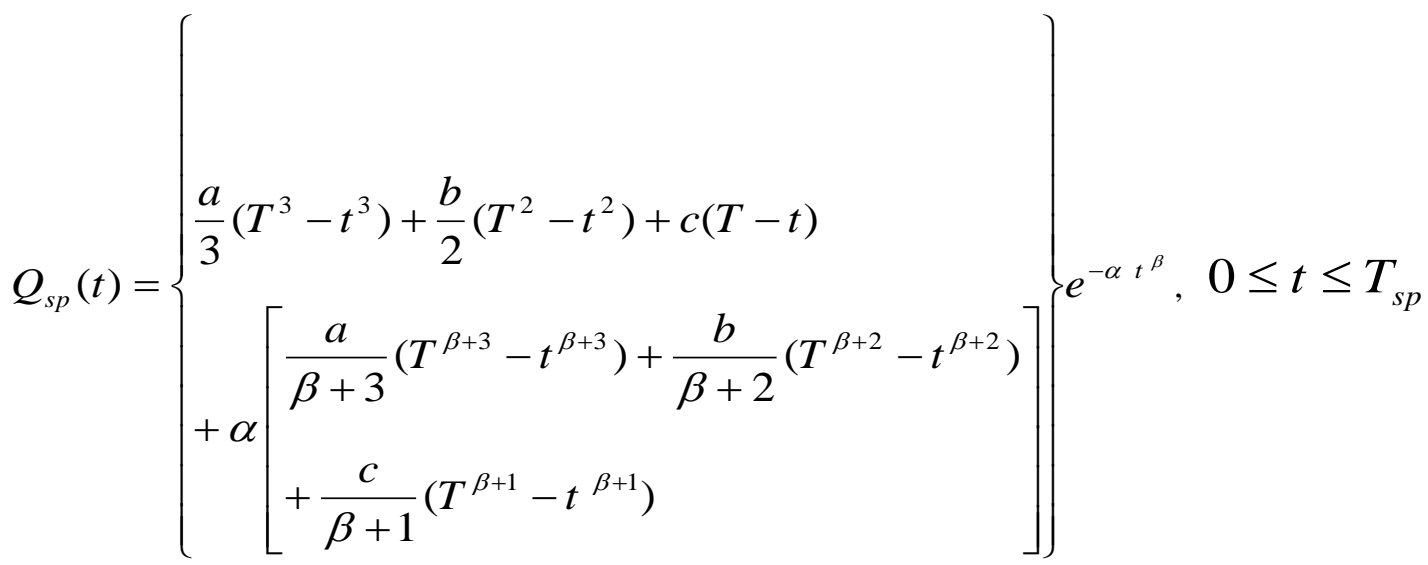

Similarly

$$
q_{s p}=\left(\frac{a T^{3}}{3}+\frac{b T^{2}}{2}+c T\right)+\alpha\left[\frac{a T^{\beta+3}}{\beta+3}+\frac{b T^{\beta+2}}{\beta+2}+\frac{c T^{\beta+1}}{\beta+1}\right]
$$

Since the price discount rate being dependent on special order let price discount rate be $\delta_{\mathrm{i}}$ in $\left(0, \mathrm{~T}_{\mathrm{sp}}\right)$ denoted by $\mathrm{TC}_{\mathrm{sp}}\left(\mathrm{T}_{\mathrm{sp}}\right)$ 


$$
\begin{aligned}
& T C_{s p l}\left(T_{s p l}\right)=h\left(1-\delta_{i}\right)\left[\left(\frac{a T_{s p l}{ }^{3}}{4}+\frac{b T_{s p l}{ }^{2}}{3}+\frac{c T_{s p l}{ }^{1}}{2}\right)+\alpha\left(\frac{a T_{s p l}{ }^{\beta+3}}{(\beta+4)}+\frac{b T_{s p l}{ }^{\beta+2}}{(\beta+3)}+\frac{c T_{s p l}{ }^{\beta+1}}{(\beta+2)}\right)\left(\frac{\beta}{(1+\beta)}\right)\right]+A_{1} \\
& +C_{2}\left(1-\delta_{i}\right)\left\{\left[\left(\frac{a T_{s p l}{ }^{2}}{3}+\frac{b T_{s p l}}{2}+c\right)+\alpha\left(\frac{a T_{s p l}{ }^{\beta+2}}{\beta+3}+\frac{b T_{s p l}{ }^{\beta+1}}{\beta+2}+\frac{c T_{s p l}{ }^{\beta}}{\beta+1}\right)\right]\right\}
\end{aligned}
$$

On the other hand, if the retailer adopts $\mathrm{Q}^{*}$ (regular order policy) in place of a large special order policy the $\mathrm{TC}\left(\right.$ Total Cost) during $\left[0, \mathrm{~T}_{\mathrm{sp}}\right]$ can be obtained by average cost approach. i.e. in the time interval $\left[0, \mathrm{~T}_{\mathrm{sp}}\right]$ the total cost of regular order is $\mathrm{TC}_{\mathrm{N}}\left(\mathrm{T}_{\mathrm{sp}}\right)$

$$
T C_{N}\left(T_{s p l}\right)=\frac{T_{s p}}{T^{*}}\left\{\begin{array}{l}
\left.h\left[\left(\frac{a T^{* 3}}{4}+\frac{b T^{* 2}}{3}+\frac{c T^{* 1}}{2}\right)+\alpha\left(\frac{a T^{* \beta+3}}{(\beta+4)}+\frac{b T^{* \beta+2}}{(\beta+3)}+\frac{c T^{* \beta+1}}{(\beta+2)}\right)\left(\frac{\beta}{(1+\beta)}\right)\right]+A_{1}\right\} \\
+C_{2}\left\{\left(\frac{a T^{* 2}}{3}+\frac{b T^{*}}{2}+c\right)+\alpha\left(\frac{a T^{* \beta+2}}{\beta+3}+\frac{b T^{* \beta+1}}{\beta+2}+\frac{c T^{* \beta}}{\beta+1}\right)\right\}
\end{array}\right\}
$$

Comparing (6) and (7) for the fixed price discount rate $\delta_{i}$, the total cost saving can be

$$
\begin{aligned}
& G_{s}\left(T_{s p l}\right)=\frac{T_{s p}}{T^{*}}\left\{\begin{array}{l}
\left.h\left[\left(\frac{a T^{* 3}}{4}+\frac{b T^{* 2}}{3}+\frac{c T^{* 1}}{2}\right)+\alpha\left(\frac{a T^{* \beta+3}}{(\beta+4)}+\frac{b T^{* \beta+2}}{(\beta+3)}+\frac{c T^{* \beta+1}}{(\beta+2)}\right)\left(\frac{\beta}{(1+\beta)}\right)\right]+A_{1}\right\} \\
+C_{2}\left\{\left(\frac{a T^{* 2}}{3}+\frac{b T^{*}}{2}+c\right)+\alpha\left(\frac{a T^{* \beta+2}}{\beta+3}+\frac{b T^{* \beta+1}}{\beta+2}+\frac{c T^{* \beta}}{\beta+1}\right)\right\}
\end{array}\right\} \\
& -h\left(1-\delta_{i}\right)\left[\left(\frac{a T_{s p l}{ }^{3}}{4}+\frac{b T_{s p l}{ }^{2}}{3}+\frac{c T_{s p l}{ }^{1}}{2}\right)+\alpha\left(\frac{a T_{s p l}{ }^{\beta+3}}{(\beta+4)}+\frac{b T_{s p l}{ }^{\beta+2}}{(\beta+3)}+\frac{c T_{s p l}{ }^{\beta+1}}{(\beta+2)}\right)\left(\frac{\beta}{(1+\beta)}\right)\right]-A_{1} \\
& -C_{2}\left(1-\delta_{i}\right)\left\{\left(\frac{a T_{s p l}{ }^{2}}{3}+\frac{b T_{s p l}}{2}+c\right)+\alpha\left(\frac{a T_{s p l}{ }^{\beta+2}}{\beta+3}+\frac{b T_{s p l}{ }^{\beta+1}}{\beta+2}+\frac{c T_{s p l}{ }^{\beta}}{\beta+1}\right)\right\}
\end{aligned}
$$

The necessary condition for a maximize $G_{s}\left(T_{s p}\right)$, is $\frac{\partial\left(G_{s}\left(T_{s p l}\right)\right)}{\partial T_{s p l}}=0$ and $\frac{\partial^{2}\left(G_{s}\left(T_{s p l}\right)\right)}{\partial T_{s p l}{ }^{2}}<0$

\section{Theoritical Results}

In this section, the optimal value of $\mathrm{T}_{\mathrm{sp}}$ that maximizes the total cost saving is determined.

Here $\delta_{i}$ denotes fixed price discount rate. For $\delta_{i}$, taking first and second derivatives of $G_{s}\left(T_{s p}\right)$ in (10) with respect to $\mathrm{T}_{\mathrm{sp}}$, gives 


$$
\begin{aligned}
& \frac{\partial\left(G_{s}\left(T_{s p}\right)\right)}{\partial T_{s p}}=\frac{1}{T^{*}}\left[h\left\{\begin{array}{l}
\left.h\left[\left(\frac{a T^{* 3}}{4}+\frac{b T^{* 2}}{3}+\frac{c T^{* 1}}{2}\right)+\alpha\left(\frac{a T^{* \beta+3}}{(\beta+4)}+\frac{b T^{* \beta+2}}{(\beta+3)}+\frac{c T^{* \beta+1}}{(\beta+2)}\right)\left(\frac{\beta}{(1+\beta)}\right)\right]+A_{1}\right] \\
+C_{2}\left\{\left(\frac{a T^{* 2}}{3}+\frac{b T^{*}}{2}+c\right)+\alpha\left(\frac{a T^{* \beta+2}}{\beta+3}+\frac{b T^{* \beta+1}}{\beta+2}+\frac{c T^{* \beta}}{\beta+1}\right)\right\}
\end{array}\right\}\right. \\
& -h\left(1-\delta_{i}\right)\left[\left(\frac{3 a T_{s p l}^{2}}{4}+\frac{2 b T_{s p l}^{1}}{3}+\frac{c}{2}\right)+\alpha\left(\frac{(\beta+3) a T_{s p l}^{\beta+2}}{(\beta+4)}+\frac{(\beta+2) b T_{s p l}^{\beta+1}}{(\beta+3)}+\frac{(\beta+1) c T_{s p l}^{\beta}}{(\beta+2)}\right)\left(\frac{\beta}{(1+\beta)}\right)\right] \\
& -C_{2}\left(1-\delta_{i}\right)\left\{\left(\frac{2 a T_{s p l}^{1}}{3}+\frac{b}{2}\right)+\alpha\left(\frac{(\beta+2) a T_{s p l}^{\beta+}}{\beta+3}+\frac{(\beta+1) b T_{s p l}^{\beta}}{\beta+2}+\frac{\beta c T_{s p l}^{\beta-1}}{\beta+1}\right)\right\}=0
\end{aligned}
$$$$
\frac{\partial^{2}\left(G_{s}\left(T_{s p}\right)\right)}{\partial T_{s p}^{2}}=-h\left(1-\delta_{i}\right)\left[\left(\frac{3 a T_{s p l}}{2}+\frac{2 b}{3}\right)+\alpha\left(\frac{(\beta+3)(\beta+2) a T_{s p l}^{\beta+1}}{(\beta+4)}+\frac{(\beta+2)\left(\beta++^{-}\right) b T_{s p l}^{\beta}}{(\beta+3)}+\frac{\beta(\beta+1) c T_{s p l}^{\beta-1}}{(\beta+2)}\right)\left(\frac{\beta}{(1+\beta)}\right)\right]_{\mathrm{To}}
$$$$
-C_{2}\left(1-\delta_{i}\right)\left\{\left(\frac{2 a}{3}\right)+\alpha\left(\frac{(\beta+2)(\beta+1) a T_{s p l}{ }^{\beta}}{\beta+3}+\frac{\beta(\beta+1) b T_{s p l}{ }^{\beta-1}}{\beta+2}+\frac{(\beta-1) \beta c T_{s p l}{ }^{\beta-2}}{\beta+1}\right)\right\}<0
$$

ensure $\mathrm{Q}^{*}<\mathrm{Q}_{\text {spl }}\left(\mathrm{i} . \mathrm{e} . \mathrm{T}^{*}<\mathrm{T}_{\text {Spl }}\right.$, where $\mathrm{T}^{*}$ can be found by solving equation (5)).

\section{Numerical Example1}

Let us consider the case when the special order occurs at the retailer's replenishment time. Considering suitable parameters viz., $\mathrm{a}=100, \mathrm{~b}=0.50 \mathrm{c}=0.20 \mathrm{~A}=100, \delta_{i}=0.1, \alpha=0.3, \beta=0.2, C_{2}=5$. From the data the optimal solutions for the regular order quantity are ascertainable and we get $\mathrm{T}^{*}=0.613, \mathrm{q}^{*}=9.9$ Besides, in the price discount offered by the supplier is studied as follows:

$$
\begin{aligned}
& \text { (i) } \mathrm{a}=100, \quad \mathrm{~b}=0.50, \mathrm{c}=0.2 \quad \quad \quad \mathrm{C}=5, \quad \alpha=0.3, \\
& \beta=0.2 \mathrm{~h}=0.1, \quad \delta=0.1, \mathrm{~A}_{1}=100 \\
& \mathrm{~T}^{*}=0.613, \mathrm{~T}_{\mathrm{sp}}=0.906, G_{s}\left(T_{s p l}\right)=31.021, \\
& \text { (ii) } \delta=0.2, \mathrm{~T}_{\mathrm{sp}}=1.107 G_{s}\left(T_{s p l}\right)=47.341, \mathrm{Q}_{\mathrm{sp}}=45.541 \\
& \text { (iii) } \delta=.25, \mathrm{~T}_{\mathrm{sp}}=1.082, G_{s}\left(T_{s p l}\right)=57.091, \mathrm{Q}_{\mathrm{sp}}=54.933 \\
& \text { (iv) } \delta=0.3, \mathrm{~T}_{\mathrm{sp}}=1.157, G_{s}\left(T_{s p l}\right)=68.198, \mathrm{Q}_{\mathrm{sp}}=67.297
\end{aligned}
$$

\section{Numerical Example2:}

On reality when we order more quantity if we consider the ordering cost is more then keeping all parameters same and increasing $A_{1}$ value the by maximizing profit we get

$$
\begin{aligned}
& \text { (i) } \mathrm{a}=100, \quad \mathrm{~b}=0.50, \mathrm{c}=0.2 \quad \quad \quad \mathrm{C}=5, \quad \alpha=0.3, \\
& \beta=0.2 \mathrm{~h}=0.1, \quad \delta=0.1, \mathrm{~A}_{1}=250 \\
& \mathrm{~T}^{*}=0.613, \mathrm{~T}_{\mathrm{sp}}=1.641, G_{s}\left(T_{s p l}\right)=31.021, \\
& \text { (ii) } \delta=0.2, \mathrm{~T}_{\mathrm{sp}}=1.837 G_{s}\left(T_{s p l}\right)=247.131, \mathrm{Q}_{\mathrm{sp}}=273.843 \\
& \text { (iii) } \delta=.25, \mathrm{~T}_{\mathrm{sp}}=1.953, G_{s}\left(T_{s p l}\right)=279.28, \mathrm{Q}_{\mathrm{sp}}=273.843 \\
& \text { (iv) } \delta=0.3, \mathrm{~T}_{\mathrm{sp}}=2.086, G_{s}\left(T_{s p l}\right)=315.861, \mathrm{Q}_{\mathrm{sp}}=403.114
\end{aligned}
$$

\section{Conclusions}

This paper suggests a decision process to assists retailers in deciding whether to adopt special order or regular order policy. For the case if special order policy is adopted, when we increase the ordering cost the optimal order quantity for the retailers is determined by maximizing the total cost saving between special and regular order during special order period. 


\section{References}

[1] Covert, R. P. and Philip, G. C., (1973). An EOQ model for items with Weibull distributed deterioration. AIIE transactions, 5, 323326.

[2] Buzacott, J. A., (1975). Economic order quantity with inflation, 1975, Operations research quarterly, Vol 26,553-558.

[3] Dave, U. and Patel, L.K., (1981). (T, $\left.\mathrm{S}_{\mathrm{i}}\right)$ - policy for inventory model for deteriorating items with time proportional demand. Journal of Operational Research Society, 32, 137-142.

[4] Misra, R. B., (1975). Optimum production lot size model for a system with deteriorating inventory. International Journal of Production Research. 13,495-505.

[5] Goyal, S.K. and Giri, B.C 'Recent trends in modeling of deteriorating inventory' (2001) European Journal of Operations research, Vol.134, pp.1-16.

[6] Jalan, A. K., Giri, R. R. and Chaudhari K.S., (1996). EOQ model for items with Weibull distribution deterioration, shortages and trended demand. International Joyrnal of System Science. 27(9), 851-855

[7] R.M.Bhandari and P.K.Sharma (2000) A single period inventory problem with quadratic demand distribution under the influence of Market policies', Eng. Science Vol. 12. No.2, pp. 117-127.

[8] Kharna, S. and Chaudhuri, K.S., (2003) 'A note on order-level inventory model for a deteriorating item with time-dependent quadratic demand' Computers and Operations research, Vol.30, pp.1901-1916.

[9] Shibshankar Sana and Chaudhary K.S., (2004) A Stock-Review EOQ Model with Stock-Dependent Demand, Quadratic Deterioration Rate', Advanced Modeling and Optimization, vol.6, No.2, pp 25-32.

[10] Mehta, Niketa, J, Shah and Nita, H.,(2006). Order-level lot size model for deteriorating items with exponentially deceasing demand. Mathematics Today, 22(1) 45-52.

[11] Soumendra Kumar Patra, Tapan Kumar Lenka and Purna Chandra Ratha (2010), An Order Level EOQ Model for Deteriorating Items in a Single Warehouse System with Price

[12] Depended Demand in Non-Linear (Quadratic) Form, International Journal of Computational and Applied Mathematics, Vol.5 No.3, pp. 277-288.

[13] Kalam, A. Samal, D. Sahu, S. K. \& Mishra, M. (2010). A Production Lot-size Inventory Model for Weibull Deteriorating Item with Quadratic Demand, Quadratic Production and Shortages, International Journal of Computer Science \& Communication, Vol. 1, No. 1, Jan. 2010, pp. 259-262

[14] Singh, S. (2011). An economic order quantity model for items having linear demand under inflation and permissible delay in payments. International Journal of Computer Applications, Vol.33, 48-55.

[15] Mohan (2014) Quadratic demand, constant deterioration, variable holding cost with shortages and salvage value, International Conference on Mathematical Sciences Proceedings, ELSEVIER, 2014, 803-806

[16] Mohan (2014) Weibull Deterioration, Quadratic Demand under Inflation, IOSR-JM Vol 10, Issue 3, $109-117$. 